\title{
Measurements of ionospheric TEC in the direction of GPS satellites and comparison with three ionospheric models
}

\author{
Giovanni Mirenna $\left({ }^{1}\right)$, Federica Riguzzi $\left({ }^{2}\right)$ and Enrico Zuccheretti $\left({ }^{2}\right)$ \\ ${ }^{1}{ }^{1}$ Istituto Elettrotecnico Nazionale, Torino, Italy \\ ${ }^{2}{ }^{2}$ Istituto Nazionale di Geofisica, Roma, Italy
}

\begin{abstract}
The IEN Galileo Ferraris uses GPS for time and frequency synchronization. To obtain high performance it is important to reduce the error due to the ionospheric time-delay in GPS measurements. Evaluations of TEC in the direction of GPS satellites, obtained from three different ionospheric models, have been compared with corresponding measurements by GPS signal.
\end{abstract}

Key words ionosphere - total electron content GPS - ionospheric delay

\section{Introduction}

In spite of its high performance, the satellitar navigation system, GPS, is subject to many errors. In particular, it is difficult to correct the delay suffered by the GPS signal crossing the ionized part of the atmosphere, i.e., the ionosphere (about 100 to $1000 \mathrm{~km}$ in height) and the plasmasphere (beyond $1000 \mathrm{~km}$ ), if a single frequency receiver is used.

At IEN a single-frequency GPS receiver has been used to synchronise its atomic clocks with those of other laboratories when a GPS satellite is visible in common view. By measuring clock offsets from GPS time (obtained from the message in the GPS signal), clocks

Mailing address: Dr. Giovanni Mirenna, Istituto Elettrotecnico Nazionale, Corso M. D'Azeglio 49, 10126 Torino, Italy; e-mail: mirennag@hotmail.com with a slight residual error (in the order of ns) can be synchronised. This residual error is mostly due to the ionospheric time-delay of the GPS signal.

IEN's research in order to reduce this error, has developed a program to evaluate ionospheric time-delay (starting from data gathered in RINEX files) for a dual-frequency receiver. Due to dispersive features in the frequency of the ionosphere, with a dual-frequency emission it is possible to correct ionospheric error, as we will describe. The results obtained at the ING (Rome) from 19th to 24th June 1996, have been compared with evaluations drawn from three different ionospheric models.

The input parameters for three models have been obtained from ionograms given every five minutes by one of the ING's ionosondes.

The models are IRI-90 (Bilitza, 1990), DGR (Radicella and Zhang, 1995) and a model obtained by reconstructing the ionospheric electron density profile on the basis of a virtual profile from ionograms for lower ionosphere, and on an Epstein contribution for the topside of F2 layer (Mirenna, 1996). 
The three models provide an evaluation of vertical TEC. This evaluation was slanted in the direction towards GPS satellite as will be described.

Ionospheric time-delay for a signal at frequency $f$ is bound to TEC along the signal path by the following:

$$
\Delta t_{\text {ion }}=\frac{40.3}{c \cdot f^{2}} \cdot \operatorname{TEC}[\mathrm{s}]
$$

where TEC is measured in TEC unit $\left(10^{16}\right.$ $\left.\mathrm{e} / \mathrm{m}^{2}\right)$. For the GPS $L 1$ carrier $(1574.42 \mathrm{MHz})$ $1 \mathrm{uTEC}$ corresponds to $0.54 \mathrm{~ns}$ of ionospheric time-delay.

This paper briefly relates how TEC evaluations were obtained from the three ionospheric models and from GPS measurements. Comparisons of the different evaluations are also reported.

\section{Evaluations of slant TEC through ionospheric models}

Most input parameters for the models such as critical frequencies and their corresponding heights, are read with a semiautomatic method implemented at ING (Zuccheretti et al., 1996). In one of the models, Polan+Epstein, the reduction to real heights up to $F 2$ peak is obtained with a program called POLAN (Titheridge, 1988).

The evaluation was drawn integrating the profile obtained from POLAN then adding the contribution of an Epstein layer to consider the topside ionosphere, invisible in ionograms. The Epstein layer is an exponential mathematical formulation to simulate the trend in ionospheric profile and it is the basis for the DGR model.

Integrating the POLAN profile and the Epstein layer profile, TEC evaluation is on the whole:

$\mathrm{TEC}=\mathrm{TEC}_{\mathrm{POLAN}}+2 \cdot k \cdot N_{m} F_{2} \cdot B_{2}\left[\mathrm{e} / \mathrm{m}^{2}\right]$

where $k$ is an empirical parameter taking into account the asymmetry between the bottomside and the topside of the $F 2$ layer ( $c f$. Radicella and Zhang, 1995), $N_{m} F_{2}$ is the peak electron density in the $F 2$ layer, $B_{2}$ is:

$$
B_{2}=\frac{0.385 \cdot N_{m} F_{2}}{(d N / d h)_{\max }}
$$

where $(d N / d h)_{\max }$ is the maximum gradient of electron density in the $F$ region.

The DGR model is based on few particular points, critical frequencies and corresponding heights in the ionogram that become fixed points for some mathematical functions approximating the electronic density profile. The Epstein function also in this case completes the topside contribute to the density profile.

IRI (International Reference Ionosphere) is the most common model describing the average quantities in the ionosphere for geomagnetically quiet conditions. This model uses the longest series of data that are the basis for electronic density profile computations and then for TEC evaluations.

Often the ionograms analysed during the four days of measurements (more than 1000) are affected by a persistent $\mathrm{E}$ sporadic layer.

Therefore traces of upper layers are often incomplete. Even if it is simple to draw the critical frequencies of upper layers, it is not so easy to watch the whole trend in the ionospheric profile necessary in the third model to calculate $(d N / d h)_{\max }$.

In any case, all three models provide an evaluation of real ionospheric TEC and they do not consider the plasmasphere. In accordance with the results obtained by Ciraolo and Spalla (1997) from IROE, Florence, it has been assumed that the plasmaspheric contribution is 3 uTEC ( \pm 1 uTEC) without either daily or seasonal perceptible variability. Therefore 3 uTEC was added to the evaluations of TEC obtained by three models in order to include plasmasphere.

At this point to obtain slant TEC in the direction of the GPS satellite, vertical TEC is multiplied by secant $Z$ of the angle of incidence with the ionosphere of the signal coming 
from the satellite given by:

$$
Z=\sec \left[\operatorname{arcsen}\left(\frac{R}{R+h} \cos \varepsilon\right)\right]
$$

where $R$ is the Earth's mean radius $(4670 \mathrm{~km})$, $h$ is the ionosphere's baricentric height (about $400 \mathrm{~km}$ ) and $\varepsilon$ is the satellite elevation calculated by the navigation message present in the GPS signal (Hofmann Wellenhof et al., 1992).

But the TEC involved in the transformation would be the one on the longitude $\lambda$ of incidence of signal with ionosphere at height $h$ (fig. 1) and not the one on the longitude $\lambda_{0}$ of the ionosonde. So it is supposed that the ionospheric features are constant in latitude, while in longitude the ionosphere is a constant structure revolving with the sun (the largest source of ionization in the atmosphere) around the Earth within a geocentric reference system. Therefore, ionospheric conditions at generic longitude $\lambda$ will be present at longitude $\lambda_{0}$ of ionosonde.

So, at time UT $t$ of GPS measurements, the corresponding time UT $t^{*}$ of the evalua- tions utilising ionospheric models for comparison is:

$$
t^{*}=t+\frac{\lambda-\lambda_{0}}{15}[\mathrm{~min}]
$$

The calculation of $\lambda$ is based on simple geometric considerations when the satellite's azimuth $\alpha$ (calculated by means of navigation message as elevation; Hofmann Wellenhof et al., 1992) and ionosonde coordinates $\left(\Phi_{0}, \lambda_{0}\right)$ are known:

$$
\begin{aligned}
& \Phi=\Phi_{0}+A \cos \alpha \\
& \lambda=\lambda_{0}+\frac{A \operatorname{sen} \alpha}{\cos \Phi_{0}}
\end{aligned}
$$

where $A$ is the geocentric angle between ionosonde (GPS receiver) and the intersection point of GPS signal with ionosphere (at longitude $\lambda$ ):

$$
A=90-\varepsilon-\operatorname{arcsen}\left(\frac{R}{R+h} \cos \varepsilon\right)
$$

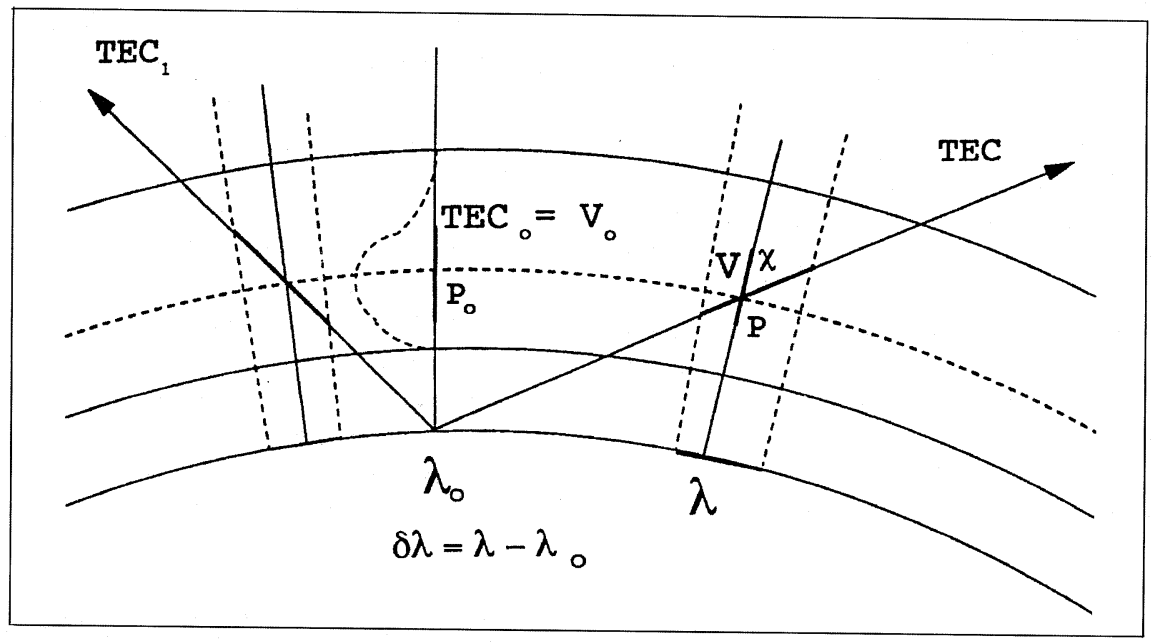

Fig. 1. Geometric model in longitude to convert estimated vertical TEC into slanting TEC in the direction of the GPS satellites. 
These approximations give errors which degrade the precision of evaluations, as to be described.

\section{Measurements of ionospheric time-delay by means of GPS}

For both GPS carriers $(L 1=1.57442 \mathrm{GHz}$ and $L 2=1.22760 \mathrm{GHz}$ ) it is possible to measure pseudoranges $R_{1}$ and $R_{2}$ and carrier phases $\phi_{1}$ and $\phi_{2}$. Measurements are usually repeated every $30 \mathrm{~s}$. Pseudorange are very noisy measurements both because of their nature and because of intentional errors in GPS (Selective Availability and Anti Spoofing; Hofmann Wellenhof, 1992). The problem with carrier phase measurements is the carrier cycle ambiguity; that is the number of full-phase cycles along the line of sight between the satellite and receiver is initially unknown.

Pseudoranges $R_{i}$ for $L i(i=1$ or 2$)$ carrier can be modelled as:

$$
R_{i}=\rho+c \Delta \delta+\Delta R_{i, \text { ion }}+\Delta R_{\text {trop }}[\mathrm{m}]
$$

where $\rho$ is the true distance receiver-satellite, $c$ is the light speed, $\Delta \delta$ is the bias between satellite atomic clock and the receiver clock - usually quartz, $\Delta R_{i, \text { ion }}$ is ionospheric delay in range unit and $\Delta R_{\text {trop }}$ is tropospheric delay.

Carrier phases $\phi_{i}$ can be modelled in range unit also as:

$$
\lambda_{i} \Phi_{i}=\rho+\lambda_{i} N_{i}+c \Delta \delta-\Delta R_{i, \text { ion }}+\Delta R_{\text {trop }}[\mathrm{m}]
$$

where $\lambda_{i}$ is the carrier wavelength and $N_{i}$ is the carrier cycle ambiguity. The minus sign in ionospheric delay is due to the different sign in the ionospheric group or phase refractive index.

In pseudorange measurements, $\Delta R_{i \text {, ion }}$ is the only term depending on signal frequency; so we can write:

$$
\begin{aligned}
& \rho=R_{1}-\Delta_{1, \text { ion }} \\
& \rho=R_{2}-\Delta_{2, \text { ion }}
\end{aligned}
$$

and using the (1.1) it is easy to deduce that:

$$
\Delta R_{1, \text { ion }}=\frac{R_{1}-R_{2}}{1-\frac{f_{1}^{2}}{f_{2}^{2}}}[\mathrm{~m}] .
$$

These and the following results were obtained by Hofmann Wellenhof et al. (1992).

Following a similar procedure for carrier phase we obtain:

$$
\Delta \Phi_{1, \text { ion }}=\frac{f_{2}^{2}}{f_{2}^{2}-f_{1}^{2}}\left(\Phi_{1}-N_{1}-\frac{f_{1}}{f_{2}}\left(\Phi_{2}-N_{2}\right)\right) \text { [cycles] }
$$

where there is the problem of not knowing $N_{1}$ and $\mathrm{N}_{2}$.

But if $N_{1}$ and $N_{2}$ are neglected and the values obtained by (3.5) are differentiated with respect to the first good datum, an extremely precise measurement of ionospheric delay variability is obtained. The first good datum is the one acquired when the satellite is over $30^{\circ}$ as minimum elevation for the first time. Subtracting this variability from (3.4), obtained data are constant except for noise which is presumably at null medium value. Working out the average of this data, its noise is removed and the «ionospheric offset» is obtained. Finally, the variability worked out using carrier phase is added to this offset.

One can observe in fig. 2 the difference in accuracy of measurements of the ionospheric time-delay in the TEC unit by using only of (3.4) or by using the algorithm described above for PRN3 satellite on 23rd June.

As a matter of fact many problems have to be worked out in order to reach the required level of uncertainty. The most important problems are cycle slip and hardware differential delays.

In fact, when the signal is tracked it can be lost for some seconds and retracking it presents a new unknown term of ambiguity. This new term cannot be removed by differentiating (3.5) as described above. But, differentiating 


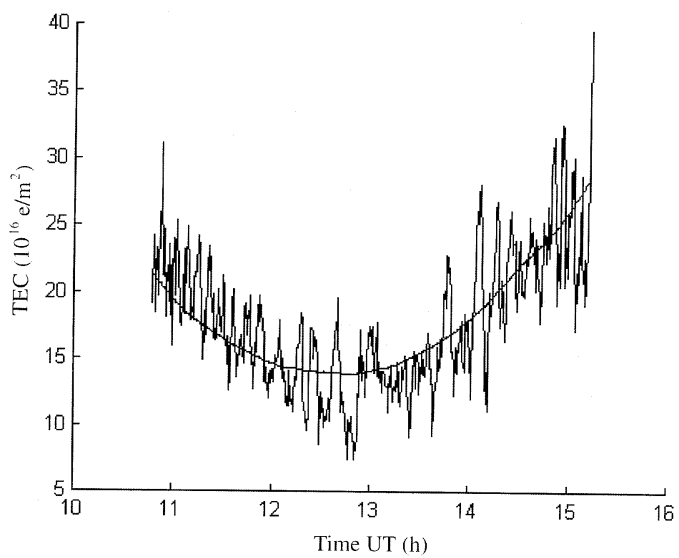

Fig. 2. Ionospheric time-delay in the TEC unit by using only pseudorange and utilizing also carrier phase for satellite PRN3 on 23rd June 1996.

(3.2) for both carriers, we obtain:

$$
\Phi_{1}-\frac{f_{1}}{f_{2}} \Phi_{2}=N_{1}-\frac{f_{1}}{f_{2}} N_{2}-\frac{\Delta R_{1, \text { ion }}}{\lambda_{1}}\left(1-\frac{f_{1}^{2}}{f_{2}^{2}}\right) \text { [cycles] }
$$

where $N_{1}$ and $N_{2}$ are constant without cycle slip, while the influence of ionospheric contribution is greatly reduced by $\left(1-\left(f_{1} / f_{2}\right)^{2}\right)$ factor by $65 \%$. Therefore the variability of (3.6) can be neglected unless a cycle slip $\Delta N_{1}$ or $\Delta N_{2}$ (according to carrier) occurs. In this case the variability $\Delta N$ of (3.6) is:

$$
\Delta N=\Delta N_{1}-\frac{f_{1}}{f_{2}} \Delta N_{2}[\text { cycles] }
$$

The minimum value 0.13 of (3.7) occurs when $\Delta N_{1}=5$ and $\Delta N_{2}=4$. Comparing $\Delta N$ with the threshold of 0.13 , cycle slips can be observed. Since delays have a parabolic trend the corresponding true value of ionospheric delay can be estimated by previous data. Actually the true threshold is not exactly 0.13 due to the ionospheric term in (3.6).
Another problem is due to hardware differential biases. Passing through satellite hardware and in particular the receiver, the signal suffers a different delay in the two GPS carriers. So by differentiating GPS measurements, a first term proportional to TEC is obtained and a second term of the same size order relative to biases is obtained. To solve this problem the solution proposed by Ciraolo (1993) was adopted based on minimization of dispersion of vertical TEC evaluations by different GPS satellites.

\section{Results and discussion}

Figure 3a-d shows data regarding satellites PRN3, PRN9, PRN15 and PRN23 on 23rd June. The solid curve is GPS data, the dashed one is DGR data, the dash-pointed one is IRI90 data and the pointed one is the third model data (Polan+Epstein).

Taking into account the azimuth of satellites (fig. 4), it can be observed that satellites to the north and mostly to the north-west almost always show overestimated measurements of ionospheric time delay according to the three models with respect to GPS measurements. On the contrary satellites to the south usually show underestimated measurements of ionospheric delay. Satellites to the west and especially to the east show minimal difference between models and GPS measurements.

From these results we can infer that considering the ionosphere invariant with latitude is limiting, especially for satellites south of the receiver. This result was predictable considering the typical ionospheric conditions varying the latitude. On the contrary, considering the ionosphere as a structure 'revolving' with the sun is a more realistic assumption.

To have an idea about the magnitude of the difference between GPS and model measurements, the absolute value of relative errors of models was calculated with respect to GPS measurements. Then the mean value and standard deviation of this quantity were calculated both daily and for the whole period (table I). 

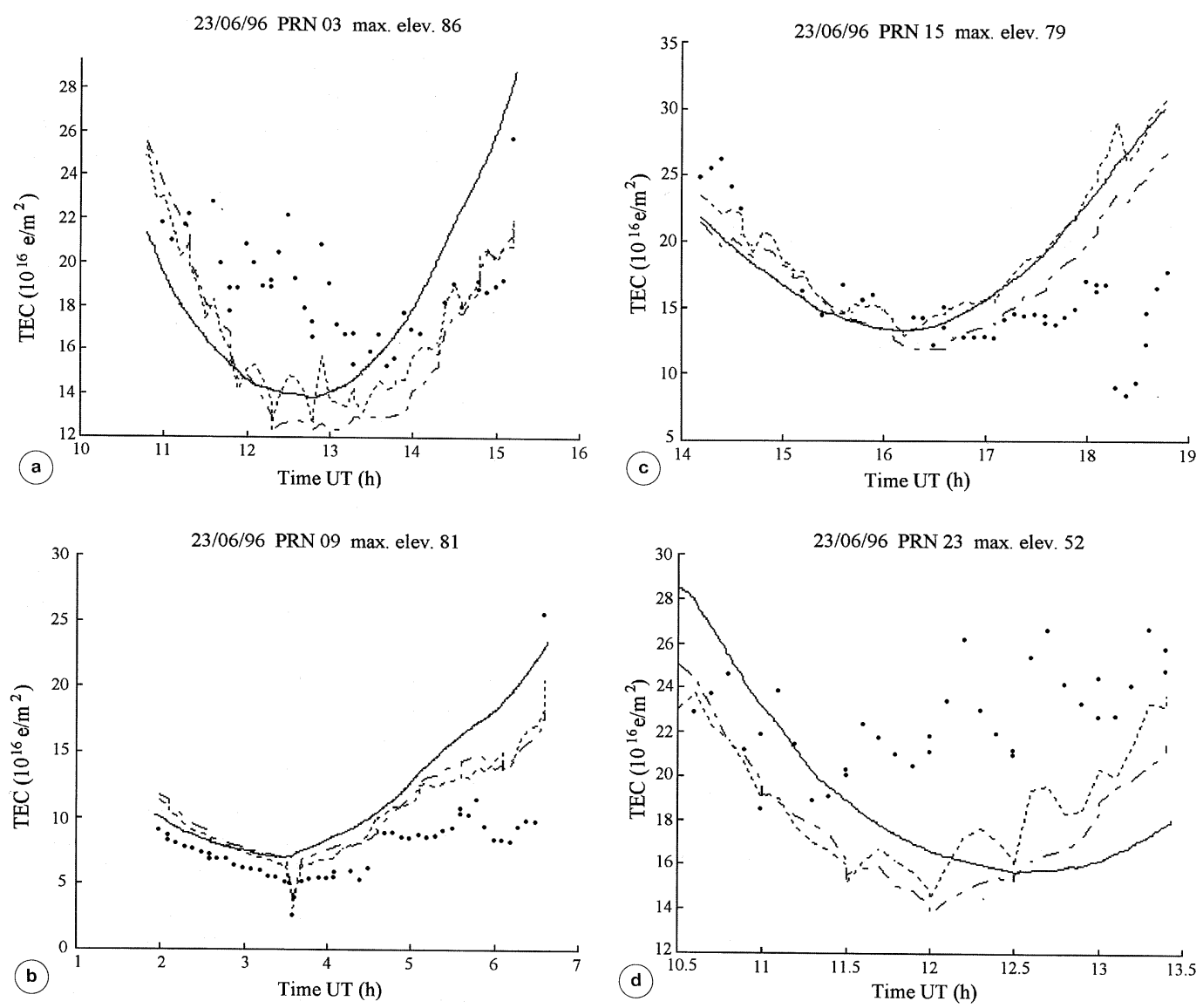

Fig. 3a-d. Ionospheric time-delay in the TEC unit about satellites PRN3 (a), PRN9 (b), PRN15 (c), PRN23 (d) on 23rd June 1996. The solid curve is GPS datum, the dashed one is DGR datum, the dash-pointed one is IRI-90 datum and the pointed one is the third model datum (Polan+Epstein).

Table I. Absolute value of the mean value and standard deviation of the difference between GPS and model measurements both daily and for whole measurement period (19th-24th June 1996).

\begin{tabular}{ccccrcc}
\hline \hline \multicolumn{7}{c}{ Total data } \\
\hline \multicolumn{2}{c}{ DGR } & \multicolumn{3}{c}{ IRI } & \multicolumn{2}{c}{ Polan+Epstein } \\
\hline & $<e_{r}>$ & $\sigma_{e r}$ & $<e_{r}>$ & $\sigma_{e r}$ & $<e_{r}>$ & $\sigma_{e r}$ \\
\hline $19 / 06 / 96$ & 16.45 & 11.06 & 13.61 & 10.30 & 23.95 & 15.78 \\
$20 / 06 / 96$ & 13.81 & 10.1 & 13.24 & 9.02 & 26.18 & 17.31 \\
$22 / 06 / 96$ & 13.11 & 12.57 & 11.84 & 11.08 & 21.31 & 16.23 \\
$23 / 06 / 96$ & 12.89 & 9.66 & 11.55 & 7.82 & 23.06 & 15.1 \\
Final & 14.17 & 10.93 & 12.59 & 9.63 & 23.66 & 16.16 \\
\hline
\end{tabular}




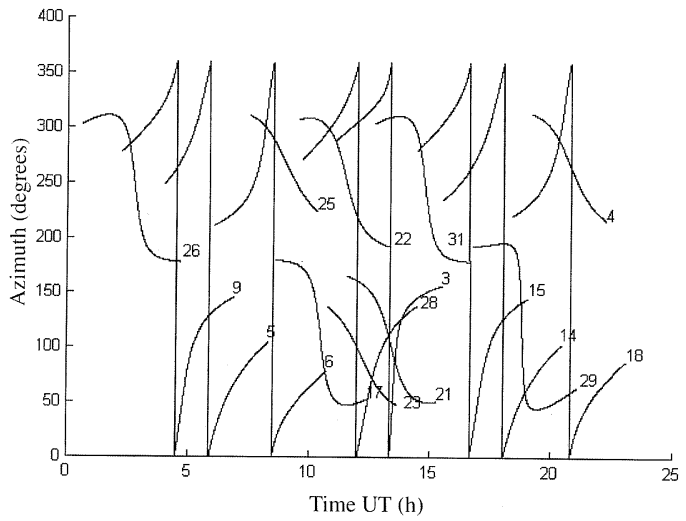

Fig. 4. Azimuth of the GPS satellites in Rome on 23rd June 1996.

\section{Conclusions}

Ionospheric time-delay measurements for GPS signals utilising pseudorange and carrier phase measurements are certainly the best ones obtained. Detecting and correcting cycle slips and estimation of hardware differential biases are still critical points. On the basis of the obtained results the maximum mistake in these measurements is estimated at about 1-2 uTEC (about $1 \mathrm{~ns}$ ) and it occurs mostly when maximum satellite elevations are low.

Evaluations performed by ionospheric models are on the contrary less satisfactory both because of several approximations passing from vertical to slant TEC, and because of the presence of a persistent E sporadic layer not al- lowing for a precise acquisition of input data for models. This last problem is mostly evident from results obtained by the third model (Polan+Epstein) which would be, theorically, the best one. In fact the Polan+Epstein model is based on the whole electronic ionospheric density profile and not only on a few characteristic points.

\section{REFERENCES}

BILITZA, D. (1990): International Reference Ionosphere 1990, NSSDC 90-92, World Data Center A Rockets \& Satellites, Greenbelt, U.S.A.

CIRAOLO, L. (1993): Evalutation of GPS L2-L1 biases and related daily TEC profiles, in Proceedings of Workshop on Modelling the Ionosphere for GPS Applications, Neustrelitz, 29-30 September 1993, 90-97.

Ciraolo, L. and P. Spalla (1997): Comparision of TEC evalutation from NNSS and GPS, Radio Sci., 32 (3), 1071-1080.

Hofmann Wellenhof, B., H. Lichtenegger and J. Collins (1992): GPS Theory and Practice (SpringerVerlag, Wien), pp. 326.

MirenNA, G. (1996): Correzioni ionosferiche per il sistema GPS, Tesi di Ingegneria delle Telecomunicazio$n i$, Politecnico, Torino.

RADICELLA, S.M. and M.-L. ZHANG (1995): The improved DGR analytical model of electron density height profile and total electron content in the ionosphere, Ann. Geofis., 38 (1), 35-41.

TITHERIDGE, J.E. (1988): The real height analysis of ionograms: a generalized formulation, Radio Sci., 23 (5), 831-849.

ZuCCheretti, E., C. BiAnCHI and G. Tutone (1996): Acquisition and off-line analysis for ionospheric data, private communication to be published by Geoinformatica.

(received February 5, 1997; accepted February 10, 1997) 